\title{
On the Periods of Biperiodic Fibonacci and Biperiodic Lucas Numbers
}

\author{
Dursun Tascı and Gul Ozkan Kızılırmak \\ Department of Mathematics, Gazi University, 06500 Ankara, Turkey \\ Correspondence should be addressed to Gul Ozkan Kızılırmak; gulozkan@gazi.edu.tr
}

Received 1 October 2016; Accepted 22 November 2016

Academic Editor: Allan C. Peterson

Copyright (c) 2016 D. Tasc1 and G. Ozkan Kizılırmak. This is an open access article distributed under the Creative Commons Attribution License, which permits unrestricted use, distribution, and reproduction in any medium, provided the original work is properly cited.

\begin{abstract}
This paper is concerned with periods of Biperiodic Fibonacci and Biperiodic Lucas sequences taken as modulo prime and prime power. By using Fermat's little theorem, quadratic reciprocity, many results are obtained.
\end{abstract}

\section{Introduction}

Fibonacci sequence and Lucas sequence are well-known sequences among integer sequences. The Fibonacci numbers satisfy the recurrence relation $F_{n}=F_{n-1}+F_{n-2}$ with the initial conditions $F_{0}=0$ and $F_{1}=1$. Binet's Formula for the Fibonacci sequence is

$$
F_{n}=\frac{\alpha^{n}-\beta^{n}}{\alpha-\beta},
$$

where $\alpha=(1+\sqrt{5}) / 2$ and $\beta=(1-\sqrt{5}) / 2$ are roots of the characteristic equation $x^{2}-x-1=0$. The positive root $\alpha$ is known as "golden ratio." Fibonacci numbers and their generalizations have many interesting properties and applications to almost every field of science and art [1-3]. In particular, Edson and Yayenie [4] introduced the Biperiodic Fibonacci sequence as follows:

$$
\begin{aligned}
& q_{0}=0, \\
& q_{1}=1, \\
& q_{n}=\left\{\begin{array}{ll}
a q_{n-1}+q_{n-2}, & \text { if } n \text { is even } \\
b q_{n-1}+q_{n-2}, & \text { if } n \text { is odd }
\end{array} \text { for } n \geq 2,\right.
\end{aligned}
$$

where $a$ and $b$ are two nonzero real numbers. We take $a$ and $b$ as integers. If we take $a=b=1$, we get the Fibonacci sequence $\{0,1,1,2,3,5,8, \ldots\}$.
If we take $a=b=2$, we get the Pell sequence $\{0,1,2,5,12,29,70, \ldots\}$. Similarly, if we take $a=b=k$, we get the $k$-Fibonacci sequence [5-8]. Binet's Formula for the Biperiodic Fibonacci sequence is given as

$$
\begin{aligned}
q_{m} & =\left(\frac{a^{1-\xi(m)}}{(a b)^{\lfloor m / 2\rfloor}}\right) \frac{\alpha^{m}-\beta^{m}}{\alpha-\beta} \\
& =\frac{1}{a^{\lfloor(m-1) / 2\rfloor} b^{\lfloor(m+1) / 2\rfloor}} \frac{\alpha^{m}-\beta^{m}}{\alpha-\beta},
\end{aligned}
$$

where $\alpha, \beta=\left(a b \pm \sqrt{a^{2} b^{2}+4 a b}\right) / 2$ are the roots of the characteristic equation $x^{2}-a b x-a b=0$ and $\xi(m)=$ $m-2\lfloor m / 2\rfloor$. Moreover,

$$
\begin{aligned}
(\alpha \beta) & =-a b, \\
(\alpha+\beta) & =a b .
\end{aligned}
$$

This sequence and its properties can be found in $[1,4]$.

Another well-known sequence is the Lucas sequence which satisfies the same recurrence relation as the Fibonacci sequence $L_{n}=L_{n-1}+L_{n-2}$ with the initial conditions $L_{0}=2$ and $L_{1}=1$. Binet's Formula for the Lucas sequence is

$$
L_{n}=\alpha^{n}+\beta^{n}
$$


where $\alpha$ and $\beta$ are defined in (1). Bilgici defined generalization of Lucas sequence similar to the Biperiodic Fibonacci sequence as follows:

$$
\begin{aligned}
& l_{0}=2, \\
& l_{1}=a, \\
& l_{n}=\left\{\begin{array}{ll}
b l_{n-1}+l_{n-2}, & \text { if } n \text { is even } \\
a l_{n-1}+l_{n-2}, & \text { if } n \text { is odd }
\end{array} \text { for } n \geq 2,\right.
\end{aligned}
$$

where $a$ and $b$ are two nonzero real numbers. We take $a$ and $b$ as integers. This sequence and other generalizations of Lucas sequence with their properties can be found in $[9,10]$. If we take $a=b=1$, we get the Lucas sequence $\{2,1,3,4$, $7,11,18, \ldots\}$. Also, if we take $a=b=k$, we get the $k$-Lucas sequence [11].

$$
\begin{aligned}
l_{n} & =\left(\frac{a^{\xi(n)}}{(a b)^{\lfloor(n+1) / 2\rfloor}}\right) \alpha^{n}+\beta^{n} \\
& =\frac{1}{a^{\lfloor n / 2\rfloor} b^{\lfloor(n+1) / 2\rfloor}}\left(\alpha^{n}+\beta^{n}\right),
\end{aligned}
$$

where $\alpha, \beta$, and $\xi$ are defined in (3).

On the other hand several researchers have made significant studies about the period of the recurrence sequences [2]. Wall [12] defined the period-length of the recurring series obtained by reducing a Fibonacci series by a modulus $m$. As an example, the Fibonacci sequence mod 3 is

$$
0,1,1,2,0,2,2,1,0,1,1, \ldots
$$

and has period 8. Vinson [3] and Robinson [13] both extended Wall's study. Moreover, they studied the Fibonacci sequence for prime moduli and showed that for primes $p \equiv 1,4$ (mod5) the period-length of the Fibonacci sequence $\bmod p$ divides $p-1$, while for primes $p \equiv 2,3(\bmod 5)$ the period-length of the Fibonacci sequence $\bmod p$ divides $2(p+$ 1).

Gupta et al. [14] give alternative proofs of this results that also use the Fibonacci matrix. They place the roots of its characteristic polynomial in an appropriate splitting fields. Renault [15] examined the behaviour of the $(a, b)$-Fibonacci sequence under a modulus.

Lucas studied the $(a, b)$-Fibonacci sequence extensively. He assigned $\Delta=a^{2}+4 b$ and deduced that if $\Delta$ is quadratic residue (that is, a nonzero perfect square) mod $p$, then $\alpha(p) \mid p-1$. If $\Delta$ is quadratic nonresidue then $\alpha(p) \mid p+1$. Also, Rogers and Campbell studied the period of the Fibonacci sequence mod $j$ [16]. They investigated the Fibonacci sequence modulo $p$ prime and then generalized to prime powers.

\section{Period of Biperiodic Fibonacci Sequence}

In this section, we investigate the Biperiodic Fibonacci sequence modulo $p$ prime and then generalize to prime powers.
Definition 1. The period of the Biperiodic Fibonacci sequence modulo a positive integer $j$ is the smallest positive integer $m$ such that

$$
\begin{array}{rlr}
q_{m} \equiv 0 & (\bmod j), \\
q_{m+1} \equiv 1 & (\bmod j) .
\end{array}
$$

By the definition above, the only members that can possibly come back to the starting point are multiples of $m$. This can be summed up in the statement that if $m$ is the period of $q_{n}(\bmod j)$, then,

$$
\begin{gathered}
q_{k} \equiv 0 \quad(\bmod j) \\
q_{k+1} \equiv 1 \quad(\bmod j) \\
\mathbb{1} \\
m \mid k,
\end{gathered}
$$

for any $k \in Z$.

Theorem 2. Let $p$ be a prime and let $n$ be a positive integer. If

$$
a \equiv 1 \quad(\bmod p)
$$

then,

$$
a^{p^{n}} \equiv 1 \quad\left(\bmod p^{n+1}\right) .
$$

We remark that the proof of the Theorem 2 can be seen in [16].

Theorem 3. Let $p$ be a prime, let $k$ be a positive integer, and let $\alpha$ and $\beta$ be the fundamental roots of the Biperiodic Fibonacci sequence. If $m$ is the period of $q_{n}(\bmod p)$,

$$
\alpha^{m p^{k-1}} \equiv \beta^{m p^{k-1}} \equiv 1 \quad\left(\bmod p^{k}\right) .
$$

Proof. For $a \neq 0$ and $p$ is a prime integer, we have

$$
q_{m}=\left(\frac{\left(a^{1-\xi(m)}\right)}{(a b)^{\lfloor m / 2\rfloor}}\right) \frac{\alpha^{m}-\beta^{m}}{\alpha-\beta} \equiv 0 \quad(\bmod p),
$$

and then

$$
\alpha^{m} \equiv \beta^{m} \quad(\bmod p) .
$$

Also we obtain

$$
\begin{aligned}
q_{m} & \equiv q_{m+1}-q_{1} \quad(\bmod p) \\
& \equiv\left(\frac{a^{1-\xi(m+1)}}{(a b)^{\lfloor(m+1) / 2\rfloor}}\right) \frac{\alpha^{m+1}-\beta^{m+1}}{\alpha-\beta}-\frac{\alpha-\beta}{\alpha-\beta} \\
& \equiv 0 \quad(\bmod p) .
\end{aligned}
$$

If $m$ is even, then

$$
q_{m} \equiv\left(\frac{1}{(a b)^{m / 2}}\right) \frac{\alpha\left(\alpha^{m}-1\right)-\beta\left(\beta^{m}-1\right)}{\alpha-\beta}
$$


From $\alpha^{m} \equiv \beta^{m}(\bmod p)$, we get

$$
q_{m} \equiv\left(\frac{1}{(a b)^{m / 2}}\right)\left(\alpha^{m}-1\right) \equiv 0 \quad(\bmod p) .
$$

Thus, $\alpha^{m} \equiv \beta^{m} \equiv 1(\bmod p)$. From Theorem 2,

$$
\alpha^{m p^{k-1}} \equiv \beta^{m p^{k-1}} \equiv 1 \quad\left(\bmod p^{k}\right) .
$$

If $m$ is odd,

$$
q_{m} \equiv\left(\frac{a}{(a b)^{(m+1) / 2}}\right)\left(\frac{\alpha^{m+1}-\beta^{m+1}}{\alpha-\beta}-\frac{\alpha-\beta}{\alpha-\beta}\right)
$$

$(\bmod p)$.

For $\alpha^{m} \equiv \beta^{m}(\bmod p)$ and $a /(a b)^{(m+1) / 2} \neq 0$,

$$
q_{m} \equiv\left(\frac{a}{(a b)^{(m+1) / 2}}\right)\left(\alpha^{m}-1\right) \equiv 0 \quad(\bmod p) .
$$

Therefore, $\alpha^{m} \equiv \beta^{m} \equiv 1(\bmod p)$. From Theorem 2,

$$
\alpha^{m p^{k-1}} \equiv \beta^{m p^{k-1}} \equiv 1 \quad(\bmod p) .
$$

Theorem 4. Let $p$ be an odd prime, let $m$ denote the period of $q_{n}(\bmod p)$, and let $\Delta=a^{2} b^{2}+4 a b$ be a nonzero quadratic residue $\bmod p$; then $m \mid p-1$.

Proof. As is known from Fermat's little theorem,

$$
\begin{aligned}
& \alpha^{p^{-1}} \equiv 1 \quad(\bmod p), \\
& \beta^{p-1} \equiv 1 \quad(\bmod p) .
\end{aligned}
$$

Thus, we have

$$
\begin{aligned}
q_{p-1} & \equiv\left(\frac{1}{a^{\lfloor(p-2) / 2\rfloor} b^{\lfloor(p-1) / 2\rfloor}}\right) \frac{\alpha^{p-1}-\beta^{p-1}}{\alpha-\beta} \equiv 0 \\
q_{p} & =\left(\frac{1}{a^{\lfloor(p-1) / 2\rfloor} b^{\lfloor p / 2\rfloor}}\right) \frac{\alpha^{p}-\beta^{p}}{\alpha-\beta} \\
& \equiv\left(\frac{1}{(a b)^{(p-1) / 2}}\right) \frac{\alpha-\beta}{\alpha-\beta} \equiv \frac{1}{(a b)^{(p-1) / 2}} \quad(\bmod p) .
\end{aligned}
$$

From $a b=-\alpha \beta$, we have

$$
(a b)^{(p-1) / 2}=\left((-\alpha \beta)^{p-1}\right)^{1 / 2} \equiv 1 \quad(\bmod p),
$$

and then

$$
q_{p} \equiv 1 \quad(\bmod p)
$$

So that

$$
\begin{aligned}
q_{p-1} \equiv 0 & (\bmod p), \\
q_{p} \equiv 1 \quad & (\bmod p) .
\end{aligned}
$$

Therefore, (10) implies that $m \mid p-1$.
Lemma 5. If $\triangle$ is a quadratic nonresidue $\bmod p$, then $(\sqrt{\triangle})^{p}=$ $-\sqrt{\triangle}$

Lemma 6. Let $\alpha$ and $\beta$ be the two roots of $x^{2}-a b x-a b=0$ in $F=F_{p^{2}} . \Delta$ is a quadratic nonresidue $\bmod p$; then $\beta^{p+1}=\alpha^{p+1}$.

Theorem 7. Let $p$ be an odd prime, let $m$ denote the period of $q_{n}(\bmod p)$, and let $\triangle$ be a quadratic nonresidue $\bmod p$; then $m \mid 2 p+2$.

Proof. From the Binomial theorem, we get $(\alpha-\beta)^{p} \equiv \alpha^{p}-$ $\beta^{p}(\bmod p)$. It follows that

$$
\begin{aligned}
q_{p} & =\left(\frac{1}{a^{\lfloor(p-1) / 2\rfloor} b^{\lfloor p / 2\rfloor}}\right) \frac{\alpha^{p}-\beta^{p}}{\alpha-\beta} \\
& =\left(\frac{1}{(a b)^{(p-1) / 2}}\right) \frac{\alpha^{p}-\beta^{p}}{\alpha-\beta} \\
& \equiv\left(\frac{1}{(a b)^{(p-1) / 2}}\right) \frac{(\alpha-\beta)^{p}}{\alpha-\beta}(\bmod p) \\
& \equiv\left(\frac{1}{(a b)^{(p-1) / 2}}\right) \frac{(\sqrt{\triangle})^{p}}{\sqrt{\triangle}}(\bmod p) \\
& \equiv\left(\frac{1}{(a b)^{(p-1) / 2}}\right) \frac{-\sqrt{\triangle}}{\sqrt{\triangle}}(\bmod p) \\
& \equiv-1 \quad(\bmod p), \\
q_{p+1} & =\left(\frac{1}{a^{\lfloor p / 2\rfloor} b^{\lfloor(p+1) / 2\rfloor}}\right) \frac{\alpha^{p+1}-\beta^{p+1}}{\alpha-\beta} \\
& =\left(\frac{1}{a^{(p-1) / 2} b^{(p+1) / 2}}\right) \frac{\alpha^{p+1}-\beta^{p+1}}{\alpha-\beta} .
\end{aligned}
$$

From Lemma 6, we obtain

$$
q_{p+1} \equiv 0 \quad(\bmod p) .
$$

Thus, $m+p+1$. Also, we have

$$
\begin{aligned}
q_{2 p+2} & =\left(\frac{1}{a^{\lfloor(2 p+1) / 2\rfloor} b^{\lfloor(2 p+2) / 2\rfloor}}\right) \frac{\alpha^{2 p+2}-\beta^{2 p+2}}{\alpha-\beta} \\
& =\left(\frac{1}{(a b)^{p}}\right) \frac{\left(\alpha^{p+1}\right)^{2}-\left(\beta^{p+1}\right)^{2}}{\alpha-\beta} \equiv 0 \quad(\bmod p),
\end{aligned}
$$

$$
\begin{aligned}
q_{2 p+3} & =\left(\frac{1}{a^{\lfloor(2 p+2) / 2\rfloor} b^{\lfloor(2 p+3) / 2\rfloor}}\right) \frac{\alpha^{2 p+3}-\beta^{2 p+3}}{\alpha-\beta} \\
& =\left(\frac{1}{(a b)^{p+1}}\right) \frac{\left(\alpha^{p+1}\right)^{2} \alpha-\left(\beta^{p+1}\right)^{2} \beta}{\alpha-\beta}
\end{aligned}
$$




$$
\begin{aligned}
& =\left(\frac{1}{(a b)^{p+1}}\left(\alpha^{p+1}\right)^{2}\right) \frac{\alpha-\beta}{\alpha-\beta} \equiv \frac{\left(\alpha^{p+1}\right)^{2}}{(a b)^{p+1}} \\
& \equiv \frac{\left(\alpha^{p+1}\right)^{2}}{(-\alpha \beta)^{p+1}} \equiv \frac{\alpha^{p+1}}{\beta^{p+1}} \equiv 1 \quad(\bmod p) .
\end{aligned}
$$

Thus, from (10), $m \mid 2 p+1$.

Theorem 8. Let $p$ be a prime, let $m$ denote the period of $q_{n}(\bmod p)$, and let $m^{\prime}$ denote the period of $q_{n}\left(\bmod p^{k}\right)$. If $m p^{k-1}$ is even then $m^{\prime} \mid m p^{k-1}$ and if $m p^{k-1}$ is odd then $m^{\prime} \mid 4 m p^{k-1}$.

Proof. We have shown that $\alpha^{m p^{k-1}} \equiv \beta^{m p^{k-1}} \equiv 1\left(\bmod p^{k}\right)$ in Theorem 3. So that

$$
\begin{aligned}
q_{m p^{k-1}} & =\left(\frac{a^{1-\xi\left(m p^{k-1}\right)}}{(a b)^{\left\lfloor m p^{k-1} / 2\right\rfloor}}\right) \frac{\alpha^{m p^{k-1}}-\beta^{m p^{k-1}}}{\alpha-\beta} \equiv 0 \\
q_{m p^{k-1}+1} & =\left(\frac{a^{1-\xi\left(m p^{k-1}+1\right)}}{(a b)^{\left\lfloor\left(m p^{k-1}+1\right) / 2\right\rfloor}}\right) \frac{\alpha^{m p^{k-1}+1}-\beta^{m p^{k-1}+1}}{\alpha-\beta} \\
& \equiv \frac{a^{1-\xi\left(m p^{k-1}+1\right)}}{(a b)^{\left.\left\lfloor m p^{k-1}+1\right) / 2\right\rfloor}}\left(\bmod p^{k}\right) .
\end{aligned}
$$

If $m p^{k-1}$ is even, then

$$
q_{m p^{k-1}+1} \equiv \frac{1}{(a b)^{m p^{k-1} / 2}} \quad\left(\bmod p^{k}\right) .
$$

From $\alpha \beta=-a b$ and by using Theorem 3, it follows that

$$
\begin{aligned}
(a b)^{m p^{k-1} / 2}=(-\alpha \beta)^{m p^{k-1} / 2}=\left((-\alpha \beta)^{m p^{k-1}}\right)^{1 / 2} & \equiv 1 \\
& \left(\bmod p^{k}\right) .
\end{aligned}
$$

Then,

$$
q_{m p^{k-1}+1} \equiv 1 \quad\left(\bmod p^{k}\right) .
$$

Thus, from (10), $m^{\prime} \mid m p^{k-1}$.

$$
\text { If } m p^{k-1} \text { is odd then }
$$

$$
\begin{aligned}
& q_{4 m p^{k-1}}=\left(\frac{a^{1-\xi\left(4 m p^{k-1}\right)}}{(a b)^{\left\lfloor m m p^{k-1} / 2\right\rfloor}}\right) \frac{\alpha^{4 m p^{k-1}}-\beta^{4 m p^{k-1}}}{\alpha-\beta} \equiv 0 \\
& q_{4 m p^{k-1}+1} \\
& \quad=\left(\frac{a^{1-\xi\left(4 m p^{k-1}+1\right)}}{(a b)^{\left\lfloor\left(4 m p^{k-1}+1\right) / 2\right\rfloor}}\right) \frac{\alpha^{4 m p^{k-1}+1}-\beta^{4 m p^{k-1}+1}}{\alpha-\beta} \\
& \equiv \frac{1}{(a b)^{2 m p^{k-1}}}\left(\bmod p^{k}\right) .
\end{aligned}
$$$$
\left(\bmod p^{k}\right)
$$

Since $\alpha \beta=-a b$ and $\alpha^{m p^{k-1}} \equiv \beta^{m p^{k-1}} \equiv 1\left(\bmod p^{k}\right)$, then,

$$
\begin{aligned}
(a b)^{2 m p^{k-1}}=(-\alpha \beta)^{2 m p^{k-1}}=\left((-\alpha \beta)^{m p^{k-1}}\right)^{2} & \equiv 1 \\
& \left(\bmod p^{k}\right),
\end{aligned}
$$

$$
q_{4 m p^{k-1}+1} \equiv 1 \quad\left(\bmod p^{k}\right) .
$$

Thus, $m^{\prime} \mid 4 m p^{k-1}$.

\section{Period of Biperiodic Lucas Sequence}

In this section, we investigate the Biperiodic Lucas sequence modulo $p$ a prime similar to Biperiodic Fibonacci sequence.

Definition 9. The period of the Biperiodic Lucas sequence modulo a positive integer $j$ is the smallest positive integer $t$ such that

$$
\begin{aligned}
l_{t} \equiv 2 & (\bmod j), \\
l_{t+1} \equiv a & (\bmod j) .
\end{aligned}
$$

For the same reasons as the Biperiodic Fibonacci sequence we have that if $t$ is the period of $l_{n}(\bmod j)$, then

$$
\begin{gathered}
l_{k} \equiv 0 \quad(\bmod j), \\
l_{k+1} \equiv 1 \quad(\bmod j) \\
\mathbb{\mathbb { I }} \\
t \mid k .
\end{gathered}
$$

for any $k \in Z$,

Theorem 10. Let $p$ be an odd prime, let $t$ denote the period of $l_{n}(\bmod p)$, and let $\triangle$ be a nonzero quadratic residue $\bmod p$; then $t \mid p-1$.

Proof. We use Fermat's little theorem to get

$$
\begin{array}{ll}
\alpha^{p-1} \equiv 1 & (\bmod p), \\
\beta^{p-1} \equiv 1 & (\bmod p),
\end{array}
$$

so

$$
\begin{aligned}
& l_{p-1}=\left(\frac{1}{a^{\lfloor(p-1) / 2\rfloor} b^{\lfloor p / 2\rfloor}}\right)\left(\alpha^{p-1}+\beta^{p-1}\right) \equiv \frac{2}{(a b)^{(p-1) / 2}} \\
& (\bmod p) .
\end{aligned}
$$

We know $(a b)^{(p-1) / 2} \equiv 1(\bmod p) ;$ thus

$$
l_{p-1} \equiv 2 \quad(\bmod p) .
$$


Also, we have

$$
\begin{aligned}
l_{p} & =\left(\frac{1}{a^{\lfloor p / 2\rfloor} b^{\lfloor(p+1) / 2\rfloor}}\right)\left(\alpha^{p}+\beta^{p}\right) \\
& \equiv\left(\frac{1}{a^{(p-1) / 2} b^{(p+1) / 2}}\right)(\alpha+\beta) \quad(\bmod p) \\
& \equiv \frac{a b}{(a b)^{(p-1) / 2}} \equiv \frac{a b}{b} \quad(\bmod p) \\
& \equiv a \quad(\bmod p) .
\end{aligned}
$$

By using (10), we get $t \mid p-1$.

Theorem 11. Let $p$ be an odd prime, let $t$ denote the period of $l_{n}(\bmod p)$, and let $\triangle$ be a quadratic nonresidue $\bmod p$; then $t \mid 2 p+2$.

Proof. From Lemma 6 and (3), we get

$$
\begin{aligned}
& l_{2 p+2}=\left(\frac{1}{a^{\lfloor(2 p+2) / 2\rfloor} b^{\lfloor(2 p+3) / 2\rfloor}}\right)\left(\alpha^{2 p+2}+\beta^{2 p+2}\right) \\
& \equiv\left(\frac{1}{(a b)^{p+1}}\right)\left(\left(\alpha^{p+1}\right)^{2}+\left(\beta^{p+1}\right)^{2}\right) \quad(\bmod p) \\
& \equiv \frac{2\left(\beta^{p+1}\right)^{2}}{(a b)^{p+1}}(\bmod p) \\
& \equiv \frac{2\left(\beta^{p+1}\right)^{2}}{\alpha^{p+1} \beta^{p+1}} \quad(\bmod p) \\
& \equiv \frac{2 \beta^{p+1}}{\alpha^{p+1}}(\bmod p) \\
& \equiv 2 \quad(\bmod p) \text {, } \\
& l_{2 p+3}=\left(\frac{1}{a^{\lfloor(2 p+3) / 2\rfloor} b^{\lfloor(2 p+4) / 2\rfloor}}\right)\left(\alpha^{2 p+3}+\beta^{2 p+3}\right) \\
& =\left(\frac{1}{a^{p+1} b^{p+2}}\right)\left(\alpha^{2 p+3}+\beta^{2 p+3}\right) \\
& =\left(\frac{1}{a^{p+1} b^{p+2}}\right)\left(\left(\alpha^{p+1}\right)^{2} \alpha+\left(\beta^{p+1}\right)^{2} \beta\right) \\
& =\frac{1}{(\alpha+\beta)\left(\alpha^{p+1}\right)^{2}}(a b)^{p+1} b \\
& =\frac{1}{(a b)\left(\alpha^{p+1}\right)^{2}}(a b)^{p+1} b=\frac{1}{a\left(\alpha^{p+1}\right)^{2}} \alpha^{p+1} \beta^{p+1} \\
& =\frac{1}{a \alpha^{p+1}} \beta^{p+1}=\frac{1}{a} \quad(\bmod p) \text {. }
\end{aligned}
$$

Thus, from (10), $m \mid 2 p+2$.

\section{References}

[1] M. Edson, S. Lewis, and O. Yayenie, “The $k$-periodic Fibonacci sequence and extended Binet's formula," Integers, vol. 11, pp. 739-751, 2011.

[2] J. Kramer and V. E. Hoggatt, Special Cases of Fibonacci Periodicity, 1972.

[3] J. Vinson, "The relation of the period modulo $m$ to the rank of apparition of $\mathrm{m}$ in the fibonacci sequence," The Fibonacci Quarterly, pp. 37-45, 1963.

[4] M. Edson and O. Yayenie, "A new generalization of Fibonacci sequence and extended Binet's formula," Integers. Electronic Journal of Combinatorial Number Theory, vol. 9, pp. 639-654, 2009.

[5] S. Falcón and Á. Plaza, "On the Fibonacci k-numbers," Chaos, Solitons \& Fractals, vol. 32, no. 5, pp. 1615-1624, 2007.

[6] S. Falcon and Á. Plaza, " $k$-Fibonacci sequences modulo $m$," Chaos, Solitons and Fractals, vol. 41, no. 1, pp. 497-504, 2009.

[7] S. Falcón and Á. Plaza, "The $k$-Fibonacci sequence and the Pascal 2-triangle," Chaos, Solitons \& Fractals, vol. 33, no. 1, pp. $38-49,2007$.

[8] S. Falcon and A. Plaza, "On $k$-Fibonacci sequences and polynomials and their derivatives," Chaos, Solutions and Fractals, vol. 39, no. 3, pp. 1005-1019, 2009.

[9] G. Bilgici, "Two generalizations of Lucas sequence," Applied Mathematics and Computation, vol. 245, pp. 526-538, 2014.

[10] G. Bilgici, "New generalizations of Fibonacci and Lucas sequences," Applied Mathematical Sciences, vol. 8, no. 29, pp. 1429-1437, 2014.

[11] S. Falcon, "On the k-Lucas numbers," International Journal of Contemporary Mathematical Sciences, vol. 21, pp. 1039-1050, 2011.

[12] D. D. Wall, "Fibonacci series modulo m," The American Mathematical Monthly, vol. 67, no. 6, pp. 525-532, 1960.

[13] D. W. Robinson, "The fibonacci matrix modulo m," The Fibonacci Quarterly, vol. 1, pp. 29-36, 1963.

[14] S. Gupta, P. Rockstroh, and F. E. Su, "Splitting fields and periods of Fibonacci sequences modulo primes," Mathematics Magazine, vol. 85, no. 2, pp. 130-135, 2012.

[15] M. Renault, "The period, rank, and order of the $(a, b)$-fibonacci sequence Mod m," Mathematics Magazine, vol. 86, no. 5, pp. 372-380, 2013.

[16] N. Rogers and C. W. Campbell, The period of the fibonacci sequence modulo $j$ [Ph.D. thesis], The University Of Arizona, Tucson, Ariz, USA, 2007.

\section{Competing Interests}

The authors declare that they have no competing interests. 


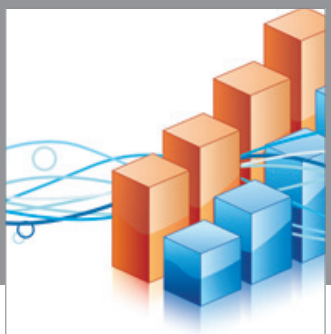

Advances in

Operations Research

vatem alat4

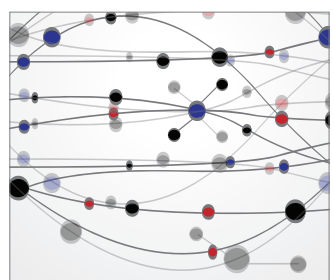

\section{The Scientific} World Journal
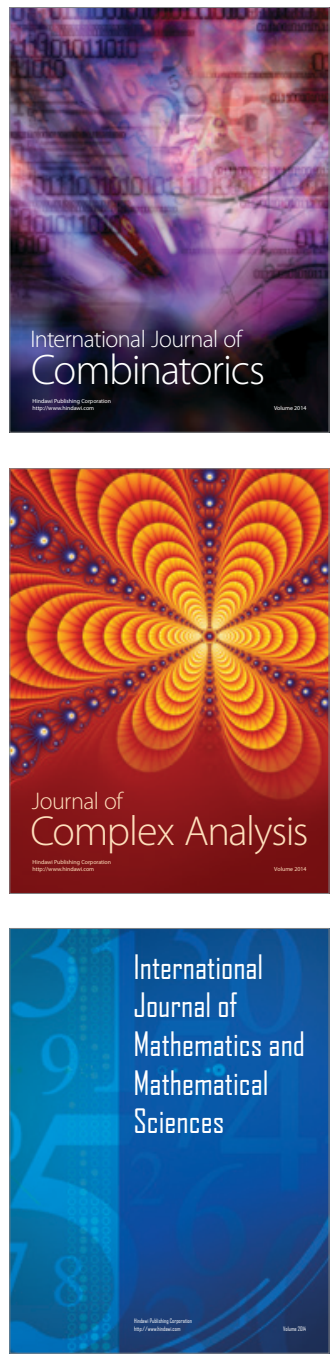
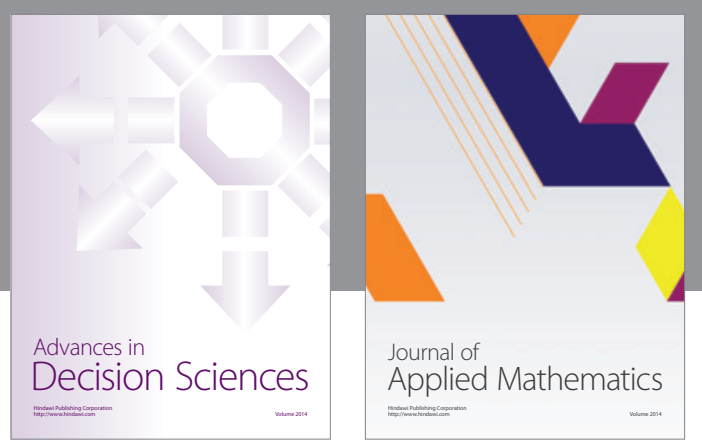

Algebra

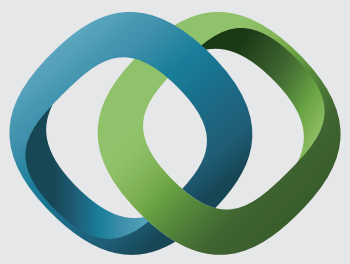

\section{Hindawi}

Submit your manuscripts at

http://www.hindawi.com
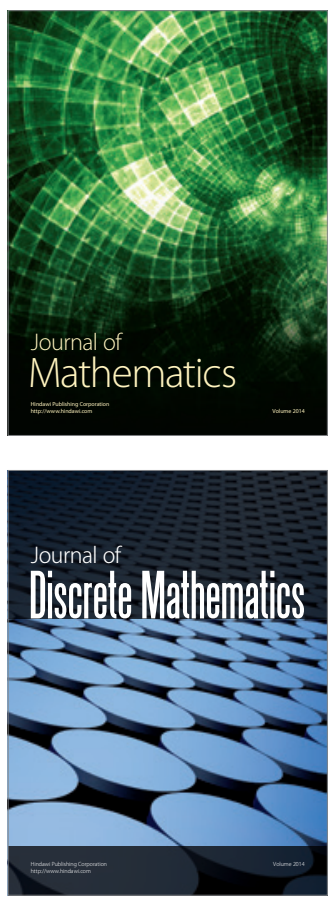

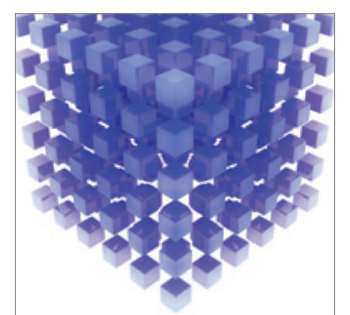

Mathematical Problems in Engineering
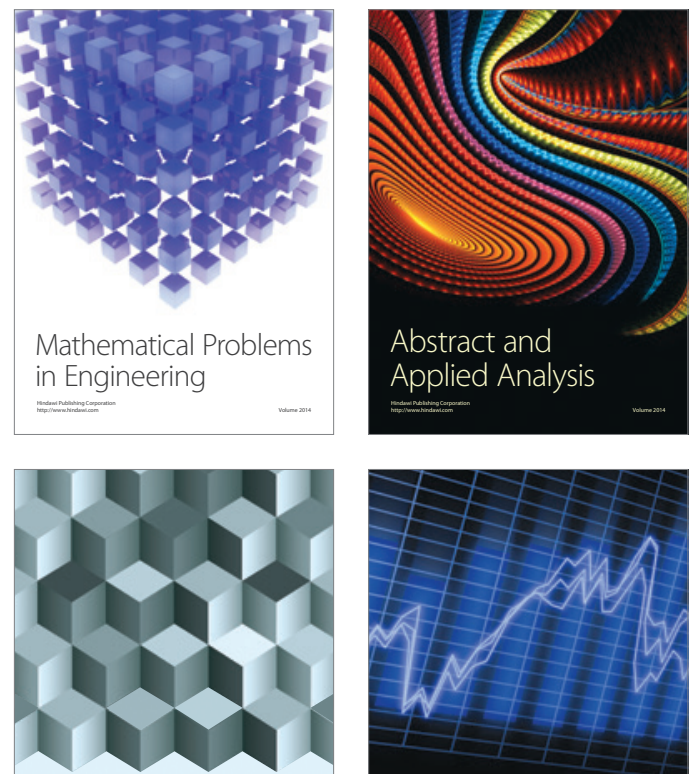

Journal of

Function Spaces

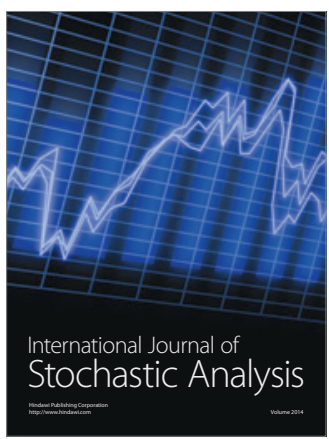

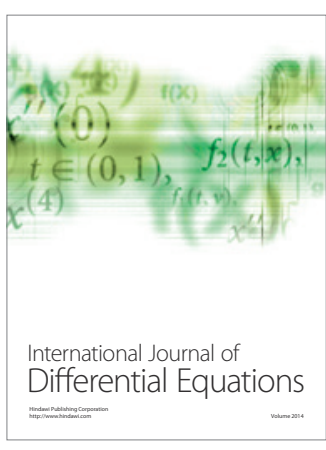
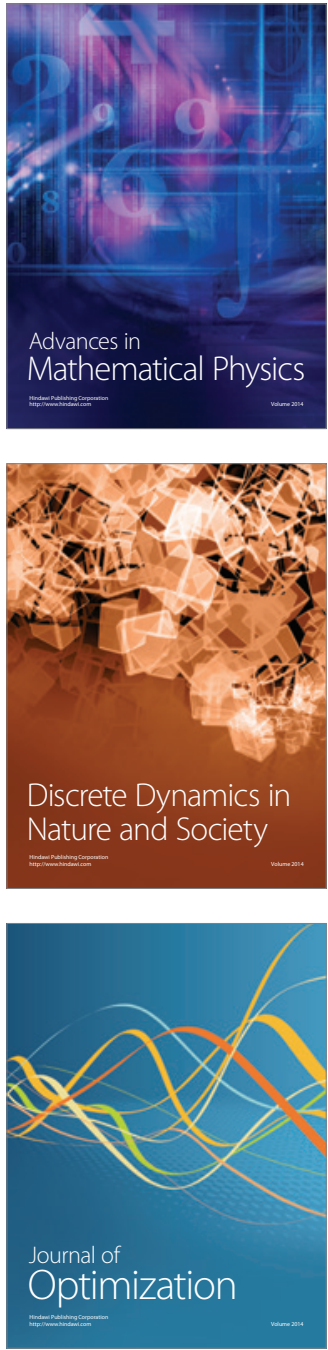